\title{
Éléments de réflexion sur les économies mésolithiques et néolithiques
}

Reflecting on mesolithic and neolithic economies

Elementos de reflexión sobre las economías mesolíticas y neoliticas

Einige Überlegungen zu den Lebens- und Umweltverhältnissen im Meso- und Neolithikum

\section{Emmanuel Ghesquière}

\section{(2) OpenEdition}

\section{Journals}

Édition électronique

URL : http://journals.openedition.org/rao/2828

DOI : $10.4000 /$ rao.2828

ISBN : 978-2-7535-5014-8

ISSN : 1775-3732

\section{Éditeur}

Presses universitaires de Rennes

\section{Édition imprimée}

Date de publication : 31 décembre 2015

Pagination : 13-24

ISBN : 978-2-7535-5012-4

ISSN : 0767-709X

\section{Référence électronique}

Emmanuel Ghesquière, "Éléments de réflexion sur les économies mésolithiques et néolithiques », Revue archéologique de l'Ouest [En ligne], 32 | 2015, mis en ligne le 28 avril 2018, consulté le 02 mars 2021. URL : http://journals.openedition.org/rao/2828 ; DOI : https://doi.org/10.4000/rao.2828 


\title{
Éléments de réflexion sur les économies mésolithiques et néolithiques
}

\author{
Reflecting on Mesolithic and Neolithic Economies
}

Emmanuel GHESQUIÈRE ${ }^{a}$

\begin{abstract}
Résumé : À partir de -15000 BP, on assiste dans le monde en général et en France en particulier à un réchauffement climatique. Les bouleversements sont spectaculaires : la moitié nord de la France, occupée alors par la steppe, est reconquise par la végétation arborée. La faune froide est remplacée par les cerfs, chevreuils et sangliers. L’homme qui pendant la période glaciaire n'occupe qu'épisodiquement le territoire se réinstalle durablement dans tous les biotopes. Jusqu’à récemment, on imaginait les populations mésolithiques comme très dispersées, vivant principalement de la prédation, comme le montrent les dernières civilisations de chasseurs-cueilleurs dans le monde qui évoluent dans des environnements désertiques (Pygmées, Inuits, Bushmen...). Il est plus que temps de mettre un terme à cette image misérabiliste du bon sauvage pour proposer celle de sociétés organisées. De nouveaux éléments de réflexion suggèrent une alimentation largement diversifiée et une sédentarité de la population, probablement accompagné d'un état de violence plus ou moins important entre groupes voisins.
\end{abstract}

Abstract: A global warming takes place around 15000 BP, with spectacular upheavals. For example the previously a steppe landscape of northern France is conquered by raised vegetation. The cold climate fauna is replaced by deer, roe deer and wild boar. All the biotopes are re-inhabited by people, who only rarely ventured into this territory during the glacial period. Until recently, we imagined Mesolithic populations as very scattered, living mainly on hunting, very similar to the last hunters-gatherers today who live in inhospitable environments (Pygmies, Luits, Bushmen). It is more than necessary to put an end to this pessimistic image of the good savage in favour of organized communities. New elements of reflection suggest a widely diversified food and a settled way of life, probably with violent clashes between neighbouring groups.

Mots clés : Mésolithique, climat, Holocène, glands, noisettes, céréales, domestication, territoire, conservation, économie, espérance de vie.

Keywords: Mesolithic, climate, Holocene, acorns, hazelnuts, cereals, domestication, territory, conservation, economy, life expectancy.

La période mésolithique, mal située entre celle des grands artistes du Paléolithique et celle des premiers agriculteurs néolithiques a toujours flotté dans une zone d'ombre. Les clichés misérabilistes atteignent même certains acteurs de la recherche traitant de la période et concernent la forêt omniprésente, une chasse et un nomadisme permanent, des groupes soumis à toutes les lois de la Nature : en un mot, des survivants de l'extrême. Ces clichés peuvent être jugés ainsi à propos des peuples vivant sous des climats hostiles, tropicaux ou glaciaires, mais semblent bien déplacés sous nos latitudes.
La tradition académique de la recherche en histoire a orienté tout son discours concernant le passé sur un progrès permanent qui s'applique principalement au niveau de vie, véritable credo justifiant tout (le PIB). Selon cette tradition, plus on recule dans le passé, plus la survie était difficile. L'époque médiévale elle-même ne serait constituée que de gueux risquant continuellement la famine quand ils ne meurent pas de peste. Il est ainsi curieux de constater qu'en Inde, les familles tirent des excédents de l'exploitation de deux hectares de terre quand il en faudrait plusieurs dizaines pour survivre dans les plaines fertiles et tempérées

a Inrap, 4 boulevard de l'Europe, 14540 Bourguebus. (emmanuel.ghesquiere@inrap.fr) 
du nord de la France! Mais restons sur des bases de sociétés pré-agricoles pour entrevoir les raisons climatiques du développement humain dans un premier temps et les raisons du non passage à une économie de production.

Le principal écueil concernant l'étude du Mésolithique, c'est le temps écoulé qui a fait disparaître les témoins autres que lithiques. Dans le meilleur des cas, les os sont conservés mais cela reste rare dans le nord de la France. En effet, après 10000 ans et sauf conditions exceptionnelles, tout ce qui reste des occupations humaines se limite à un tas de silex. Le $\mathrm{xxI}^{\mathrm{e}}$ siècle ne sera-t-il quand à lui représenté que par des fragments de porcelaine? Les archéologues du futur dirontils alors que le $\mathrm{XxI}^{\mathrm{e}}$ siècle était une période misérabiliste? Cette vision ne semble adaptée ni au Mésolithique, ni aux autres périodes que l'on est susceptible de reconnaître par l'archéologie. La misère n'est pas identifiable au contraire de l'opulence. Le Mésolithique, malgré des témoins ténus constitués presque uniquement par les outils de pierre taillé, est probablement une période opulente où l'homme possède tout ce que son époque lui propose. Guerres et mauvaises années existent et représentent probablement des épiphénomènes récurrents, mais qui ne remettent pas en cause une autonomie alimentaire basée sur une multiplicité de sources d'alimentation et une mobilité de l'ensemble de la popula- tion. La genèse décrit cette période comme le paradis (Ge 2), où l'homme n'a qu'à se baisser pour ramasser sa nourriture; ce qui est vrai pour un pays partiellement sec comme Israël sera d'autant plus vrai pour l'Europe occidentale tempérée.

\section{ENVIRONNEMENT}

L'Holocène correspond au dernier interstade glaciaire, dans lequel nous nous trouvons encore. Le climat se réchauffe à partir de - 15000 BP, d'abord de manière chaotique alternant brusquement réchauffements et refroidissements (Grootes et al., 1993), pour atteindre rapidement des températures sensiblement identiques aux actuelles vers -9500 (Stuiver et al., 2000). La différence entre climat glaciaire et climat actuel n'est que de $6^{\circ} \mathrm{C}$ de température moyenne en globalité, avec cependant des différences importantes suivant la latitude, de $15^{\circ} \mathrm{C}$ au Groenland à $2{ }^{\circ} \mathrm{C}$ dans le sud de l'Europe (Peteet 2000). De la même façon, quelques degrés de plus qu'actuellement nous séparent des fougères géantes du Carbonifère.

Au Mésolithique, la rapide remontée des températures a bouleversé tout l'écosystème (fig. 1). La flore représentée (pour les terres libérées des glaces) par de la steppe va voir une couverture arborée se développer (bouleaux, pins,

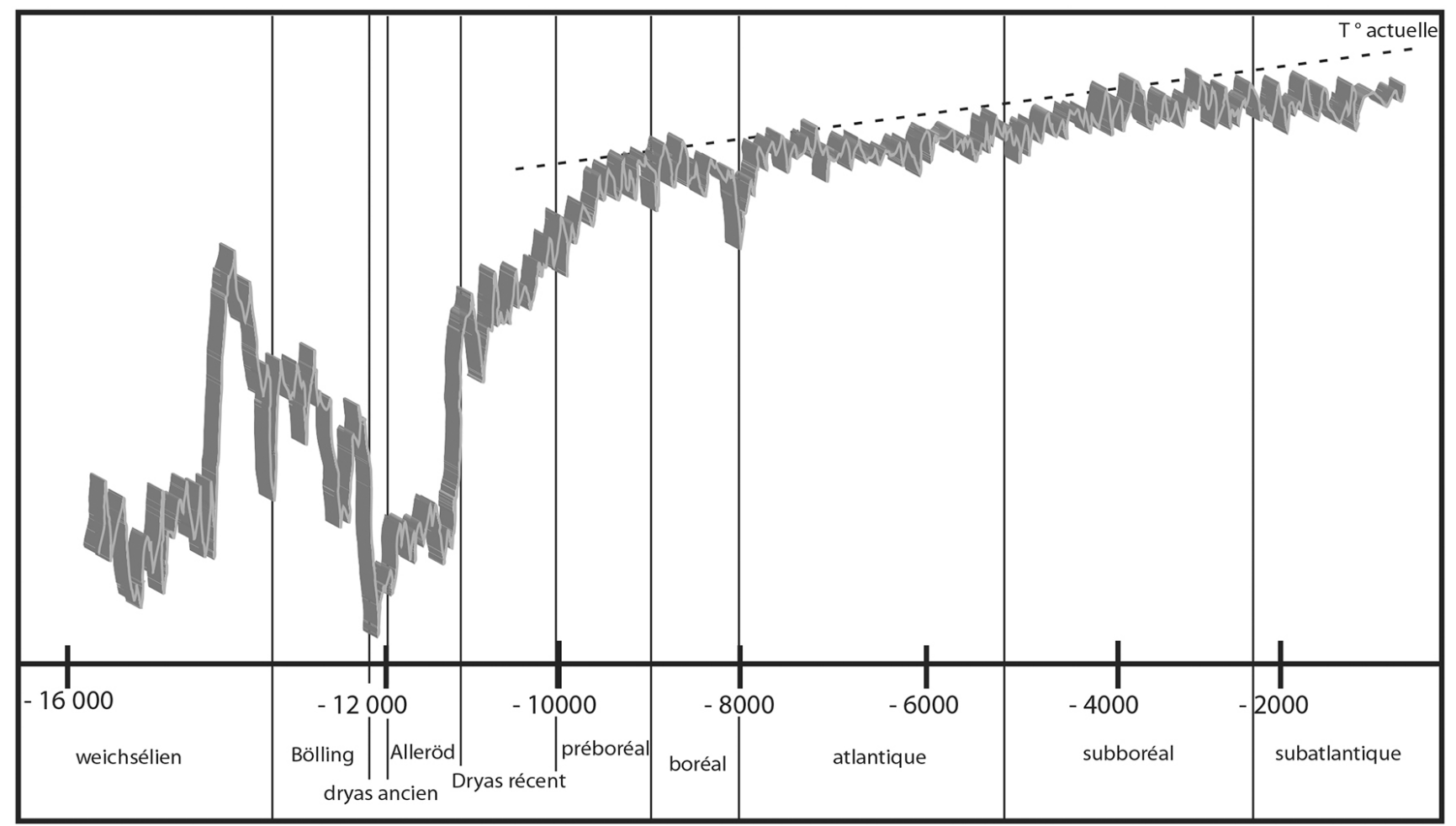

Figure 1 : Courbe simplifiée en perspective du climat à l'Holocène. Contrairement aux idées reçues du Xxi ${ }^{\mathrm{e}}$ siècle, plus il fait chaud, plus les conditions générales sont propices au développement humain à l'échelle de la planète. Mais où se situe la limite?

Figure 1: Simplified graph of the Holocene. Contrary to the popular belief of the $21^{\text {st }}$ century, the hotter it gets, the more the general conditions are conducive to human development. But where does the limit lie? 
noisetiers et chênes...) puis envahir le paysage. La faune (comestible) composée de grands troupeaux de chevaux, bisons, rennes et mammouths va disparaitre vers le nord de l'Eurasie, remplacée par des espèces tempérées se suffisant d'un environnement mi-forestier (cerfs, chevreuils, sanglier), mais vivant en petites hardes. L'homme suivra ces nouvelles espèces animales pour reconquérir le territoire.

Cette image d'Épinal connait toutefois tant de nuances que nous ne sommes pas loin de faire sauter complètement le cadre. En effet, à la période glaciaire, il semble que certaines vallées abritées (vallée de l'Erve, Mayenne, Barbier et Visset, 2000) aient connu un environnement arboré partiel, facilitant lors du réchauffement de multiples recolonisations forestières. A contrario, jusque assez tard, certaines vallées très exposées (Haute vallée de la Seine?) ont pu voir la persistance d'une végétation steppique maintenant sur place une faune glaciaire : la grotte de Gouy datée du Bölling ${ }^{1}$ (Bordes, 1974) et sa célèbre frise de chevaux, celle d'Orival voisine et sa probable gravure de proboscidien (info D. Cliquet) ou encore le locus 28704 du site d'Alizay (étude en cours), daté du début du Préboréal entre 9900 et $9300 \mathrm{cal} \mathrm{BC}$, dont l'assemblage faunique est représenté par un bois de renne et des restes de bisons.

Enfin, le point le plus discutable est l'extension réelle de la forêt. Tous les sondages polliniques le prouvent : au Mésolithique, la forêt est omniprésente (70\% des taxons identifiés dans les prélèvements). Cependant, les milieux d'observation de ces pollens sont tous identiques. Il s'agit des basses vallées qui ont enregistré ces séquences, et jamais des plateaux qui constituent l'essentiel du paysage. Pourquoi alors ne pas reconnaitre que de très larges secteurs de l'Europe mésolithique n'ont jamais connu de couvert forestier, sous des types de climats très différents, comme l'intérieur de l'Espagne, l'intérieur de la Norvège, la Hongrie, l'Écosse ou la façade Atlantique/Manche/Mer du Nord, au contraire d'autres secteurs ou la forêt a été et reste très présente (France continentale, Allemagne, Italie, Angleterre...), dans les secteurs actuellement densément occupés et industrialisés (Île-de-France par exemple). L'enregistrement des chablis et trous d'arbres, souvent très déficitaires sur les plateaux normands va dans ce sens (fig. 2), dès que l'on s'écarte de quelques centaines de mètres des couverts forestiers actuels, qui semblent inchangés depuis au moins le Moyen Âge, sauf épi-phénomènes de reforestations (Basse vallée de la Seine...) ou de déforestations industrielles (forêt de Sauxmesnil, Manche...).

1. Gif A $92346: 12050 \pm 130$ BP.

2. Beta $317897: 9890 \pm 40$ BP, Beta $333640: 9960 \pm 40$ BP, Beta $322721: 9610 \pm 50 \mathrm{BP}$, Beta $333638: 10100 \pm 40 \mathrm{BP}$

\section{2. ÉCONOMIE}

L'Holocène correspond à une révolution dans l'ensemble du monde. Elle a pu jouer un rôle néfaste pour les environnements tropicaux déjà chauds (Sahara par exemple), mais elle a été largement bénéfique sous nos climats froids.

Dans le secteur qui correspond sensiblement au Kurdistan, le phénomène de révolution néolithique a consisté dans un premier temps en une appropriation des denrées alimentaires sauvages, avant leur préservation, leur multiplication, leur amélioration et enfin leur culture raisonnée, sur une durée de plusieurs millénaires. En Europe occidentale, le rôle des céréales a pu être joué par des fruits d'arbres, glands et plus sûrement noisettes au nord de la Loire. Comme ces plantes ne sont pas annuelles mais persistantes, le recours à leur culture n'est pas indispensable comme pour les céréales, même si un entretien augmente la récolte. La domestication animale, forme de soumission de l'homme à l'animal, comme de l'animal à l'homme (Lestel, 2009), constitue la véritable innovation des néolithiques par rapport aux sociétés de chasseurs cueilleurs occidentales. Davantage que la viande, elle apporte lait et fibres textiles. L'absence en Europe de l'Ouest d'espèces indigènes de bêtes à fibres textiles a pu jouer un rôle dans l'absence de domestication animale (à l'exception du chien), car suidés et bovidés locaux auraient également pu être soumis à l'homme.

Quelle que soit la période archéologique considérée, les noisettes constituent en France le fruit le plus fréquemment rencontré, ce qui semble paradoxal pour un produit non cultivé, mais les capacités de conservation des autres produits (céréales en particulier) sont moindres. Toujours est-il que cela atteste de l'usage de ce fruit, sinon comme base de l'alimentation, au moins comme complément toutes périodes confondues. Le gland ne présente pas les mêmes facultés de conservation. Sa coquille mince ne charbonne pas et est facilement détruite par le feu. Il faut pour sa conservation les mêmes conditions que pour les grains de céréales, une calcination incomplète, facilitée au moins par une des méthodes de traitement alimentaire de ce fruit (torréfaction partielle). Ces deux arbres (noisetier et chêne) pourraient jouer un rôle majeur dans le développement des populations mésolithiques en France. Leur omniprésence dans le paysage (deux arbres sur trois sont des noisetiers ou des chênes) assurent des possibilités de ramassage et de stockage excluant toute contrainte. La collecte de ces denrées a laissé dans notre vocabulaire le synonyme de «ne rien faire ", ce qui est suffisamment éloquent. L'importance de ces deux fruits que sont la noisette et le gland ne doit pas faire oublier la masse énorme de produits potentiellement comestibles en France (châtaignes, faînes de hêtre, orties...) dont certains 


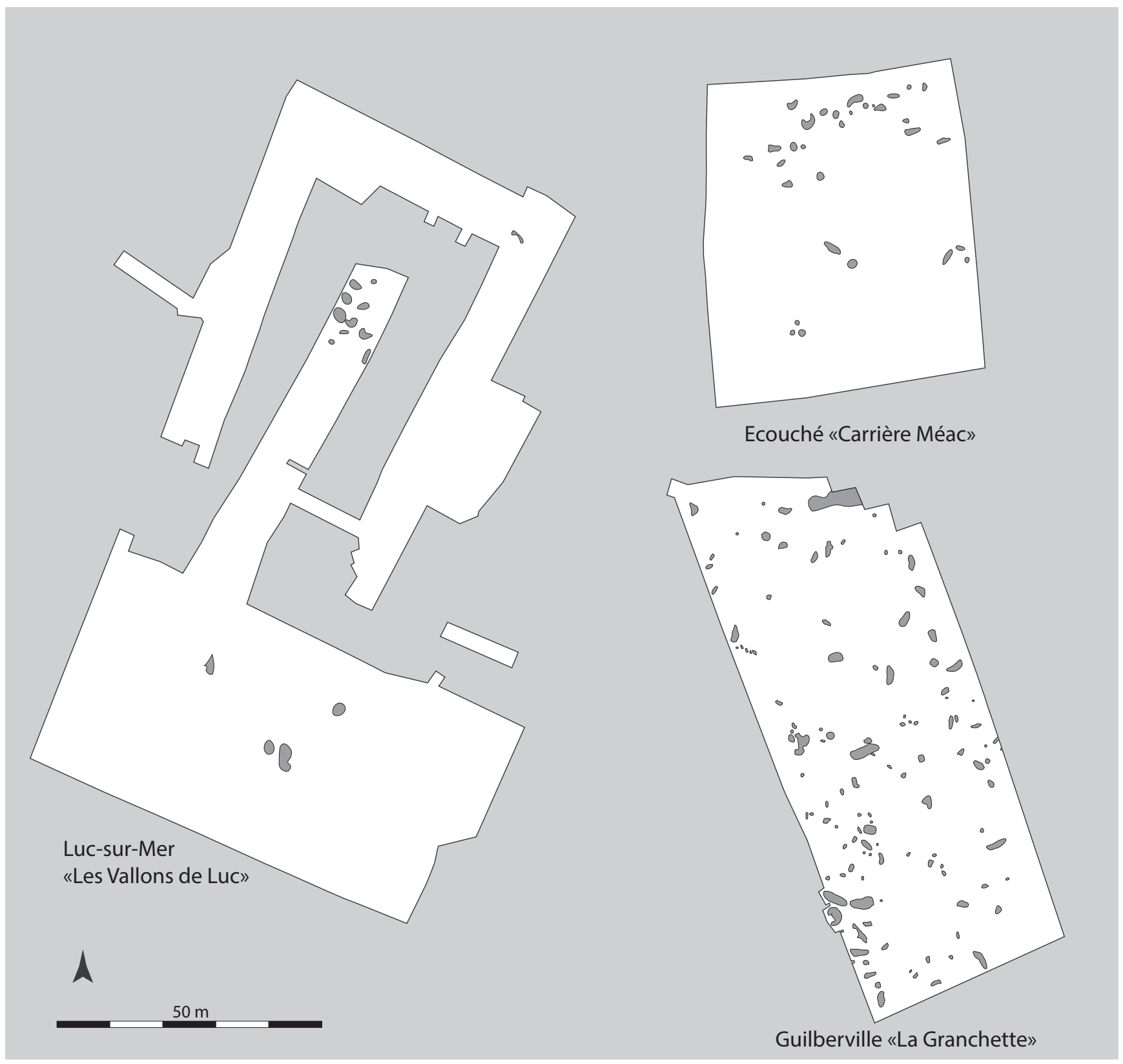

Figure 2 : Répartition des chablis sur trois sites normands. À droite en haut, le site d'Écouché « Carrière Méac " (61, Marcigny, DFS inédit), où les empreintes d'arbres se limitent à un " bosquet " au nord de l'emprise; à droite en bas, le site de Guilberville " La Granchette " (50, Ghesquière et Marcigny, 2001), en contexte probable d'ancienne forêt; à gauche le site de Luc-sur-Mer (14, Marcigny, DFS inédit) où les empreintes fossiles d'arbres sont presque inexistantes, trahissant une forêt holocène au moins très dispersée sinon inexistante dans cette plaine côtière (mer à $25 \mathrm{~km}$ au Mésolithique).

Figure 2: Distribution of tree holes on three Norman sites. At the top right, the site of Écouche "Career MEAC" (61, Marcigny unpublished DFS), where the tree holes are limited to a "copse" to the north of the site; bottom right, the site of Guilberville "The Granchette" (50, Ghesquiere and Marcigny, 2001), with a probable ancient forest context; left, the site of Luc-sur-Mer (14, Marcigny unpublished DFS) where the tree hole fossils are almost non-existent, indicating a dispersed or non-existent Holocene forest at least highly dispersed if not non-existent on the coastal plain (coastline $25 \mathrm{~km}$ away during the Mesolithic.

ont pu jouer un rôle important dans l'alimentation, en particulier aux périodes de soudure.

Le passage d'une cueillette et consommation instantanée à un stockage de masse et une consommation différée consti- tue une autre étape intellectuelle. Cette étape semble au moins atteinte dès le début de l'Holocène, comme l'attestent les récolteurs de blé sauvage du Proche-Orient, mais pourrait tout simplement être inhérente à Homo sapiens sapiens. La 
constitution de stocks de nourriture pour assurer l'alimentation durant la mauvaise saison (qui peut être sèche, froide ou humide) est la base de la survie humaine dans toutes les zones géographiques où les saisons sont marquées (zones tempérées ou froides). Seule la bande intertropicale peut se passer de stocks avec des groupes humains mobiles qui exploitent des biotopes toujours identiques, chauds et plus ou moins humides (indiens d'Amazonie, pygmées), mais même dans ces secteurs, des groupes de sédentaires/stockeurs ont existé à la période pré-coloniale (Rostain, 2011).

Dans la mesure où une partie importante de leur alimentation est végétale et qu'elle fait l'objet de stockage, les populations ne peuvent plus être mobiles et au moins durant la saison froide, entre la récolte de septembreoctobre et le début du printemps, les populations doivent se concentrer à proximité de leurs réserves. Or, la consommation humaine peut être estimée à $0,5 \mathrm{~kg}$ par personne et par jour, selon les modélisations sub-actuelles (équivalent blé/bouillies, 2000 calories/jour), si cette consommation constitue la base de l'alimentation (mais pas la totalité de l'alimentation). Pour un groupe humain d'une cinquantaine de personnes, un peu plus de neuf tonnes de glands ou de noisettes est requise pour l'année (moitié moins juste pour la saison froide). Ce volume important nécessitait des capacités de stockage et une certaine sédentarité du groupe humain, même si une partie pouvait être stockée hors des zones d'habitation ${ }^{3}$.

En ce qui concerne les différents produits de base, il faut tenir compte du fait que l'usage du gland dans l'alimentation est plus approprié en contexte méditerranéen où les espèces sont plus douces, se passant pratiquement de préparation (lessivage, torréfaction...) pour certains d'entre eux. C'est ainsi qu'il constituait la base de l'alimentation des chasseurs-cueilleurs californiens (Kroeber, 1968) et est encore en usage au Maroc et en Espagne. La noisette est beaucoup plus appropriée comme base alimentaire dans le nord de la France. Mais même sur cette base, on se doit de tenir compte de l'énorme potentiel alimentaire du gland au nord de la Loire, comme le montrent les nombreux restes carbonisés en contexte néolithique découvert en Normandie (Val-de-Reuil, Billard et al., 1995; Alizay, Aubry, rapport de diagnostic 2008, inédit), qui attestent d'une perduration de sa consommation. Le pignon de pin (parasol) et la châtaigne, troisième et quatrième fruit permettant de constituer un support alimentaire de base par son abondance, sont réservés au Mésolithique de la côte méditerranéenne.

3. On pense en particulier aux fosses cylindriques dont plusieurs centaines ont été mises en évidence en Champagne (Achard-Corompt et al., à paraître).

\section{TERritoire NÉCESSAIRE À L'ALIMENTATION VÉGÉTALE (FIG. 3)}

Il est important dans ce cadre d'évaluer le territoire nécessaire à la cueillette et donc à l'alimentation du groupe, et de comparer ces chiffres à un mode d'alimentation basé sur l'agriculture. Les principales différences des deux systèmes reposant pour le Mésolithique sur une exploitation verticale des denrées (sur arbres/arbustes) et pour les agriculteurs sur une exploitation horizontale, dans le cadre de champs de céréales ou de légumineuses. Il est difficile d'estimer la surface nécessaire pour la récolte mésolithique car celle-ci n'est pas délimitée dans l'espace et que l'exploitation repose probablement sur les individus/arbres les plus prolifiques (variables d'une année sur l'autre) ou ceux présentant telle ou telle appréciation gustative. On peut cependant considérer que cinq à dix hectares de zones boisées constituent une réserve nettement suffisante à assurer la base de l'alimentation végétale d'un groupe de cinquante personnes, considérant une fourchette basse de production de dix kilos de glands par chêne et cinq cents kilos de noisettes à l'hectare de noisetier (Barbaza, 1999), ces deux arbres formant la majorité des espèces présentes. Dans le cadre de pratiques agricoles de type archaïque, c'est-à-dire une première année sur brûlis et les récoltes suivantes avec fumage des terres (fumiers, cendres, terreaux), voire une agriculture sur limons de débordements, une surface de 4 à 8 hectares suffit à l'alimentation d'un groupe équivalent (Anderson, 1992; Bogaard, 2004) ${ }^{4}$. Il faut également tenir compte d'une rotation ou une alternance des cultures (céréales/légumineuses?) possible après plusieurs récoltes, ce qui n'est pas nécessaire avec les arbres. La différence de surface entre les deux modes d'acquisition des denrées végétales de base reste sur un modèle relativement équivalent, qui peut être limitée à la proximité immédiate de l'habitat, dans un cas comme dans l'autre. À moins de remodeler complètement les chiffres de densité de population envisagée à ces époques, en aucun cas l'exploitation des ressources végétales de base [sauvages ou domestiques] n'a pu entraîner une « crise du sol » entre communautés du Mésolithique et du Néolithique, d'autant plus que les biotopes concernés, forestier ou semi-forestier pour les premiers, ouverts pour les seconds, ne se recoupent que très partiellement.

4. D'autant plus que le fléau qu'est le lapin n'est pas encore présent en France. 


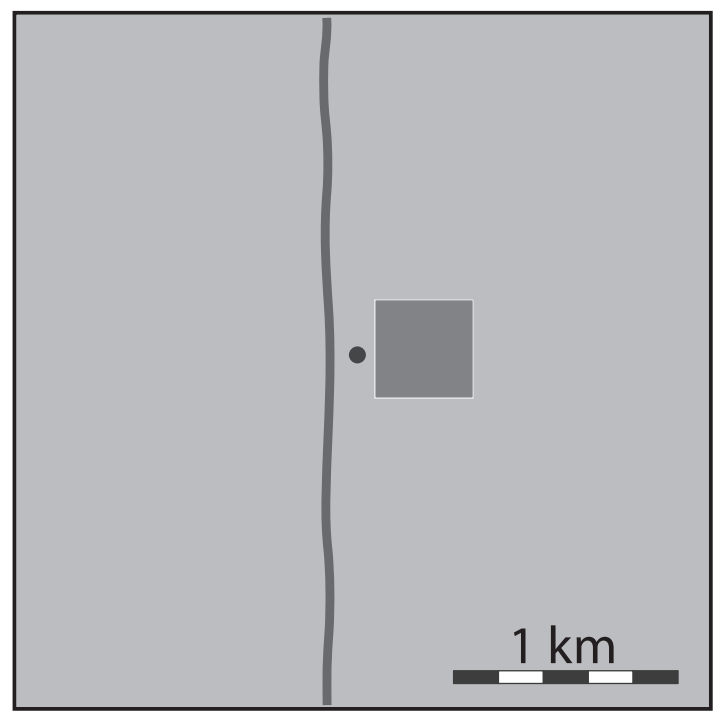

Mésolithique (village 50 personnes)

\section{TERritoires NÉCESSAIRES À L'ALIMENTATION CARNÉE (FIG. 3)}

Ces territoires s'avèrent très variables entre communautés du Mésolithique et du Néolithique. On sait qu'actuellement, la surface par bovidé est de l'ordre de 1 à 1,5 hectare de prairie, pour assurer l'alimentation sur l'année. Pour des ovicapridés rustiques de petite taille (moutons d'Ouessant...), ce chiffre peut monter à 20 pour la même surface. Le cochon peut quant à lui s'accommoder d'une surface réduite à la condition que l'homme lui apporte sa nourriture, mais cela oblige alors à multiplier les structures de stockage pour l'alimentation animale, alors même que le cheptel correspond à une forme de stockage, vivant bien sûr. D'autre part, un contexte de prairie n'est pas nécessaire à l'alimentation des troupeaux, qui s'accommodent aussi bien de friches, taillis ou même forêts. Seul l'accès à un point d'eau, naturel ou artificiel est indispensable au bétail, au moins tous les deux jours. Une surface assez réduite va donc être indispensable aux populations néolithiques pour assurer le développement de leur cheptel. En restant sur la base d'un groupe de cinquante personnes, une vingtaine de bovidés, autant de suidés et quatre-vingts ovicapridés suffisent à assurer une alimentation carnée sinon quotidienne, au moins régulière et à assurer la survie en cas de stress alimentaire ${ }^{5}$; le reste des besoins ou envies est assuré par la chasse, restreinte certes, mais toujours active. Ces animaux vont donc occuper une place d'une quarantaine d'hectares de prairies, le double ou le triple en milieu de taillis ou de forêts ${ }^{6}$.

5. Ainsi qu'à assurer les besoins en fumage des sols agricoles.

6. comme ce qui se passe encore aujourd'hui dans les forêts corses où vaches et cochons divaguent, avec un foulard comme marque de propriétaire.

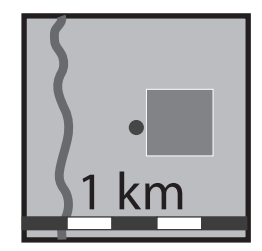

1 hectare

alimentation végétale

alimentation carnée
Néolithique (50 pers.)
Figure 3 : Chorème illustrant une estimation des surfaces nécessaires en zone tempérée non littorale d'autosuffisance alimentaire de groupes mésolithique et néolithique.

Figure 3: Chorème illustrating an estimate of the area needed in a non-coastal temperate zone for food self-sufficiency of Mesolithic and Neolithic groups.
Les territoires nécessaires à la chasse au Mésolithique sont également possibles à évaluer, en restant sur la base des quatre espèces principales de consommation, respectivement : cerf, sanglier, chevreuil et aurochs, dont l'avantage par rapport au cheptel néolithique est que leur alimentation ne se recoupe que partiellement, ce qui leur permet un usage alimentaire des mêmes terroirs. Des problèmes de comparaison vont se poser à propos des animaux sauvages beaucoup plus robustes que ceux du Néolithique; de même pour le cerf, dont le volume comestible est bien supérieur à celui d'un ovicapridé. Un ratio a donc été établi permettant de faire coïncider le poids de viande. Pour le Néolithique, un cheptel de vingt bovidés $(350 \mathrm{~kg})$, vingt cochons $(50 \mathrm{~kg})$ et quatre-vingts moutons/chèvres $(15 \mathrm{~kg})$ correspond à $11600 \mathrm{~kg}$, avec la possibilité de consommation d'un tiers par an, soit quatre tonnes (entre 40 et 100 hectares occupés). Pour le Mésolithique, une population de quarante cerfs, quarante sangliers, dix aurochs et trente chevreuils permet une chasse de onze cerfs $(90 \mathrm{~kg})$, douze sangliers (70 kg), trois aurochs $(600 \mathrm{~kg})$ et huit chevreuils $(25 \mathrm{~kg})$, soit quatre tonnes prélevées. Ces chiffres restent tout à fait théoriques et ne reflètent que des ratio sans autre fondement que d'offrir un modèle théorique de comparaison des espaces voués à l'alimentation carnée, pour une consommation là encore très aléatoire de vingt grammes de viande par personne et par jour; ratio qui permet une autonomie alimentaire à long terme si elle intervient en complément d'une base alimentaire végétale.

Dans le cas du Mésolithique, on se heurte rapidement à la difficulté de calcul du nombre d'animaux à l'hectare. De très nombreux facteurs font varier ces paramètres quant aux ressources alimentaires propres à chaque terroir, comme 
par exemple la destruction éventuelle d'une partie des prédateurs par l'homme. Les quelques chiffres que l'on peut obtenir pour le cerf consistent en des approximations : à Grimes Graves au Néolithique, où l'exploitation des cerfs est évoquée (mais réfutée par les auteurs français; Maigrot 2003), avec dix cerfs au kilomètre carré (Clutton-Brock, 1984), jusque des cas extrêmes, insulaires, où la densité est montée à 125 cerfs au kilomètre carré. La situation actuelle dans les forêts du Jura sans prédation est de deux cerfs par kilomètre carré (chiffres ONF) et la réalité mésolithique probablement encore tout autre. Il est clair toutefois que l'alimentation carnée des populations mésolithiques devait nécessiter un territoire beaucoup plus vaste que celui des populations du Néolithique, dans une fourchette probable de cinq cents à trois mille hectares, pour un groupe constitué d'une cinquantaine de personnes. Cette surface permet le développement d'une faune à vocation alimentaire suffisante, mais reste largement supérieure aux besoins de survie de la population humaine basée sur l'alimentation végétale.

\section{LA CONSERVATION DES DENRÉES}

La question du stockage des denrées ne pose guère de problèmes techniques aux populations pré-agricoles. Trois méthodes principales ont pu être utilisées dans le cadre qui nous intéresse ici. La conservation sèche, au vent, qui s'applique aussi bien aux poissons et aux mollusques qu'aux graines telles que glands ou noisettes. Le fumage s'applique essentiellement aux ressources halieutiques, chair ou œufs de poissons et coquillages. Ce principe de conservation est utilisé pour gérer les importantes pêches saisonnières au saumon (Californie par exemple; Testart, 1982), pour lesquelles la quantité de poisson pêché coïncide uniquement avec la capacité du groupe à traiter celui-ci pour la conservation. Sa préparation qui consiste à lever les filets et à les fumer est en effet assez longue et dispendieuse en temps/homme. Les traces archéologiques de telles pratiques correspondent à des foyers, généralement empierrés et dont l'accumulation sur les mêmes sites pourrait être un indice d'utilisation d'une telle activité. La présence d'arêtes de poisson dans un contexte " éloigné " de leur lieu de récolte peut également être un marqueur de la conservation de ces ressources (Philibert, 2002). Sans avoir aucune certitude, on peut évoquer le site de l'Essart à Poitiers, en bordure de rivière, où de nombreux foyers liés à une industrie moyennement riche correspondant pour chacun des locus à une occupation " temporaire " (de 10 à 30 armatures suivant les locus) pourraient évoquer de telles pratiques (Marchand, 2009).

La conservation en milieu fermé prend plusieurs formes : hors sol sec, en fosse sèche ou en fosse humide. La méthode $\mathrm{du}$ " hors sol sec » est utilisée pour les grains en climat sec, mais est également adaptée à un climat tempéré, d'autant plus si la conservation s'effectue à l'intérieur des habitations. Le stockage s'effectue dans des récipients en cuir, bois ou vannerie, parfois de très grande taille comme l'indiquent des exemples californiens de stockage de glands de un mètre de diamètre et pouvant contenir quatre cents litres de fruits. Cette méthode multiforme a pu être utilisée au Mésolithique, mais elle a la particularité de ne laisser aucune trace archéologique. Le principe de confinement dans une structure en creux (en "fosse sèche ») vise à obtenir un milieu où l'humidité est la plus limitée possible, de même que l'oxygène. Jusqu'à il y a quelques années, de telles structures étaient pratiquement inconnues en France à l'exception notable du site d'Auneau (Verjux, 2000). Depuis lors, la découverte de plusieurs centaines de fosses cylindriques en Champagne et Picardie (Achard-Corompt et al., à paraitre; Hénon et al., 2013), datées par ${ }^{14} \mathrm{C}$ du Premier Mésolithique, viennent remettre en cause nos certitudes sur les creusements mésolithiques, peut-être beaucoup plus fréquents qu'on ne le pensait, mais ignorées jusque-là car situées en dehors des habitats. Leur fonction n'est toutefois pas acquise, partagée entre diverses hypothèses (piège, silo?). À Auneau, l'attribution de ces fosses à des structures de stockage, placées éventuellement à l'abri de structures couvertes, repose sur leur volume et leur répétition (Verjux, 2004).

La dernière forme de conservation, en "fosse humide", oblige à noyer le produit afin d'éliminer l'apport d'oxygène et éviter la dégradation du produit. Peu fréquente, elle est cependant utilisée en Californie du nord par certaines tribus pour le stockage des glands. Elle permet en même temps l'élimination des tanins contenus dans le fruit. La structure mise au jour à Ronai (fig. 4), creusée dans un limon très argileux et au remplissage hydromorphe, datée du Mésolithique récent (Ghesquière, 2010), pourrait correspondre à une telle structure de stockage humide. Sa capacité de mille litres permettrait l'alimentation d'un groupe de trois personnes durant une année.

\section{6. ÉCONOMIE LITHIQUE}

À aucun autre moment de son " histoire " l'Homme n'a été moins dépendant de la matière première de son outillage qu’au Mésolithique. En effet, au Paléolithique supérieur, les populations très mobiles exploitent des silex sur des centaines de kilomètres (Kilby, 2011); au Néolithique, elles sont obligées de creuser des mines et aux âges des métaux, elles organisent le transport du minerai sur une partie de l'Europe. Le Mésolithique quant à lui se contente d'une matière première locale ou disponible dans un rayon de 

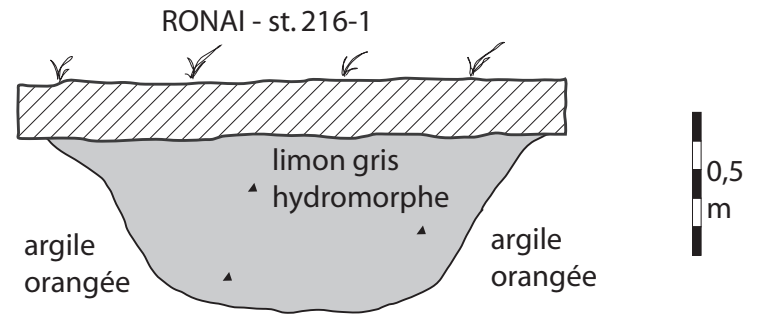

AUNEAU - Fosse $\mathrm{n}^{\circ} 7$

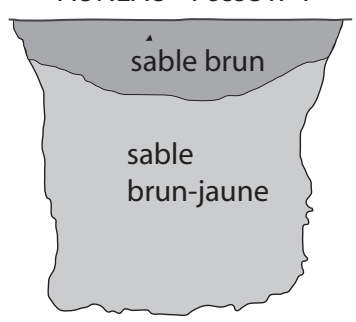

Figure 4 : Formes de fosses mésolithiques attribuées par hypothèse au stockage de denrées alimentaires (Ronai, à gauche, Ghesquière, 2010, et Auneau, à droite, Verjux, 2004).

Figure 4: forms of probable Mesolithic storage pits (left: Ronai, Ghesquière 2010, and right: Auneau, Verjux 2004). quelques dizaines de kilomètres, correspondant à son territoire de chasse ou à différents camps de base (été/hiver?). L'usage d'un très bon silex est possible lorsque celui-ci affleure (vallée de la Seine par exemple), mais le recours aux roches de substitution (quartz, ultramylonites, phtanites...) est fréquent lorsque le silex est absent, comme dans le Massif armoricain. L'industrie est alors irrégulière mais néanmoins tout à fait adaptée aux besoins. Ce détachement vis-à-vis de tout élément extérieur à leur « territoire » est une des caractéristiques de cette période, forme d'adaptation extrême au milieu (Ghesquière, en préparation). Cela donne l'image de sociétés très repliées sur elles-mêmes, avec probablement des conflits avec les groupes alentours. Cette autosuffisance et ce repli seront de sérieux handicaps à se défendre collectivement lors des périodes d'expansions territoriales (groupe Rhin-Meuse-Schelde au VIII ${ }^{e}$ millénaire, agriculteurs néolithiques au VI ${ }^{e}$ millénaire).

\section{7. ÉCONOMIE CÉRAMIQUE}

Les paniers, probablement omniprésents à l'époque, sont absents dans les faits car non conservés. Les très rares vestiges découverts en milieu humide en Europe attestent leur présence, comme sur le site de Noyen-sur-Seine (fig. 5). Cet artisanat permet une grande variété de formes et de décors. En témoignent par exemple les paniers des indiens chasseurs cueilleurs californiens, agrémentés d'ailleurs de grands récipients en bois. Ces objets témoignent à tout le moins de possibilités de stockage de divers types de produits qui peuvent être alimentaires aussi bien que de pièces d'habillement. Devant la rareté des sites humides, on en reste davantage aux suppositions, comme d'ailleurs pour l'ensemble de l'outillage en bois et même une partie de l'outillage en os. La question de la céramique, comme substitut aux paniers, pouvant aller sur le feu mais plus fragile, pose un problème

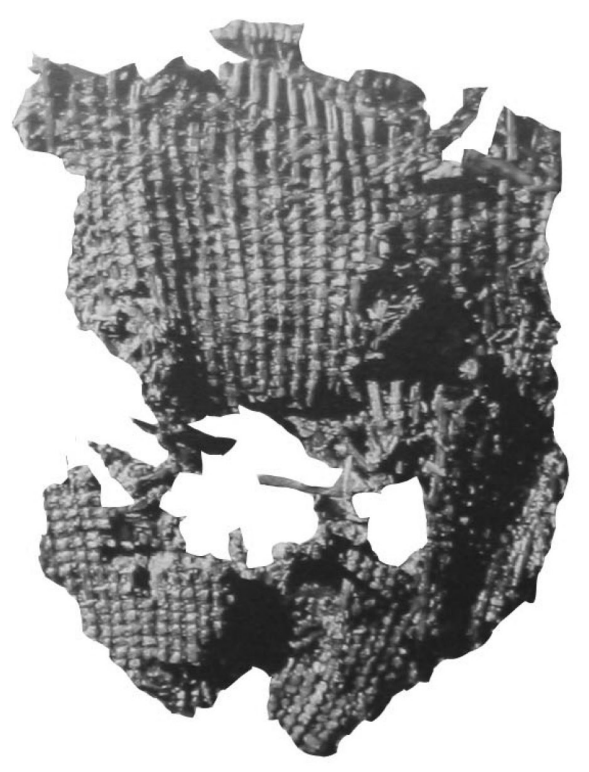

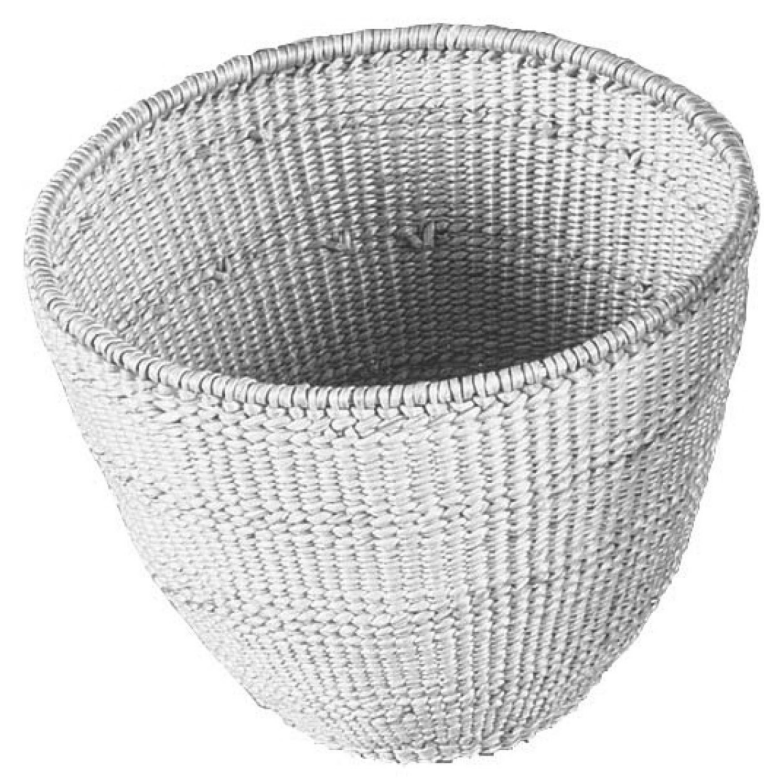

Figure 5 : Fragment de panier mésolithique moyen du site de Noyen-sur-Seine et reconstitution. Cliché D. Mordant, CG 77). Figure 5: Fragment of a Middle Mesolithic basket from Noyen-sur-Seine and reconstitution. 
plus aigu. Elle apparait comme un facteur clair de sédentarisation et est absent dans le Néolithique initial anatolien au PPNA et PPNB. Cette absence en Anatolie constitue un blocage pour une partie de la communauté scientifique qui considère leur impossibilité dans le Mésolithique français. Pourtant, la céramique est bien attestée chez les chasseurs cueilleurs d'Asie, d'Europe du Nord (Vanmontfort et al., 2010), ainsi que nombre de chasseurs cueilleurs d'Amérique (fig. 6). Sans être totalement admise, la présence de céramique de la Hoguette (mais peut-être aussi du Limbourg?), plus ancienne dans quelques secteurs que la colonisation rubanée, peut être le témoin de mésolithiques à céramique en France du Nord (Manen, 1997; Manen et Mazurié de Keroualin, 2003; Ghesquière et Aubry, 2013).

\section{8. ÉCONOMIE DE LA MORT}

Les différences principales entre populations néolithiques et mésolithiques, dans la France des $\mathrm{VI}^{\mathrm{e}}$ et $\mathrm{V}^{\mathrm{e}}$ millénaire, reposent sur une natalité beaucoup plus importante de la population néolithique, liée selon toute vraisemblance à un baby-boom permanent (Bocquet-Appel, 2009), permettant une augmentation rapide de la population (doublement à chaque génération ?). Cette expansion a permis aux agriculteurs de s'étendre rapidement sur l'ensemble du territoire français. Cette expansion liée à un apport calorique important du fait de la consommation massive de céréales s'est ajoutée à une mortalité bien supérieure à celle des chasseurscueilleurs, principalement due à l'apparition et à la multiplication des maladies infectieuses issues de la proximité avec les animaux domestiques (Zammit, 2005). Ces événements mortifères auraient entraîné, comme on le constate à partir de l'étude de plusieurs nécropoles, une diminution importante de l'espérance de vie et une pyramide des âges très décalée vers le bas. Cette faiblesse du nombre d'individus âgés n'a toutefois pas d'incidence sur le taux de natalité des populations, les naissances étant le fait des individus jeunes.

On constate à partir de l'étude des sépultures que les populations mésolithiques sont à l'abri de la plupart des causes de décès post-infantiles et présentent une espérance de vie assez importante, proches de populations primitives actuelles ayant accès à une médecine moderne de prévention et soin des maladies épidémiques $(20 \%$ de la population entre 50 et 60 ans; fig. 7). En contrepartie, leur régime faiblement calorique n'a pas permis à ces populations de développer un taux de natalité permettant de concurrencer l'expansion des populations néolithiques, dont la durée de vie plus faible assurait une forte proportion de jeunes. Cette différentiation dans la pyramide des âges a sans aucun doute participé à la supériorité des populations d'agriculteurs lors de leur conquête du continent européen. La propagation
Figure 6 : (Voir planche couleur I) Chasseurscueilleurs céramisés en Europe (d'après VanMontfort et al., 2010; Mazurié de Keroualin, 2003; Crombé, 2010 et Gronenborn, 2010), en Amérique du Nord (d'après Driver and Massey, 1957) et dans l'ensemble de l'Eurasie (d'après Jordan et Zvelebil, 2010 et Jordan et Zvelebil [éd.], 2010). En Europe, l'apparition d'une céramique chez les Mésolithiques est bien acceptée pour le nord du continent (Danemark, Hollande et Belgique) entre 5300 et 4000 , mais est encore très controversée pour la France (La Hoguette et Limbourg).

Figure 6: (See colour plate I) Pottery producing hunter-gathers in Europe (from VanMontfort et al., 2010, Mazurié de Keroualin 2003 Crombé and Gronenborn 2010), North America (after Driver and Massey 1957) and throughout the Eurasian (after Jordan and Zvelebil 2010 and Jordan and Zvelebil [ed.], 2010). In Europe, the theory of the emergence of pottery amongst the Mesolithic peoples is accepted for the north of the continent (Denmark, Holland and Belgium) between 5300 and 4000 but is still very controversial in France (La Hoguette and Limburg).
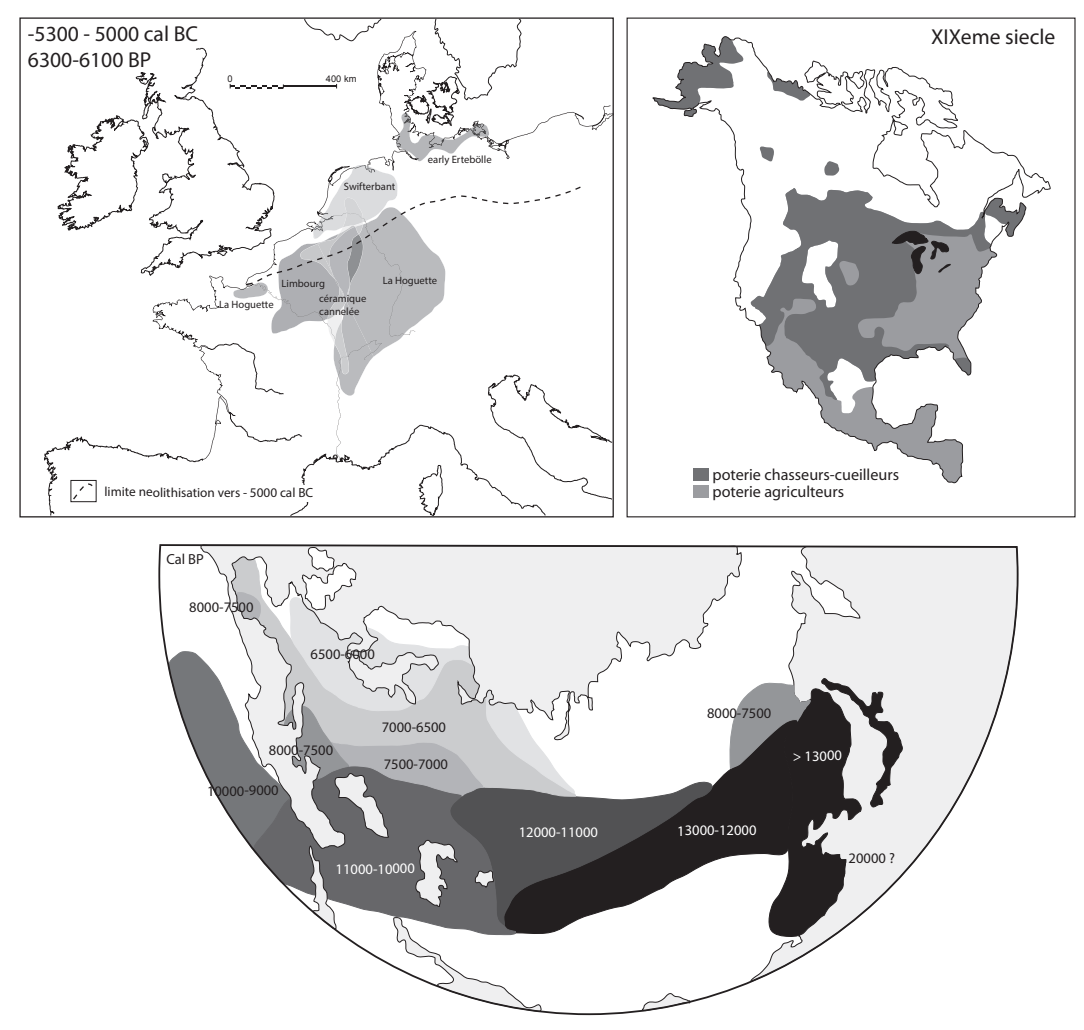


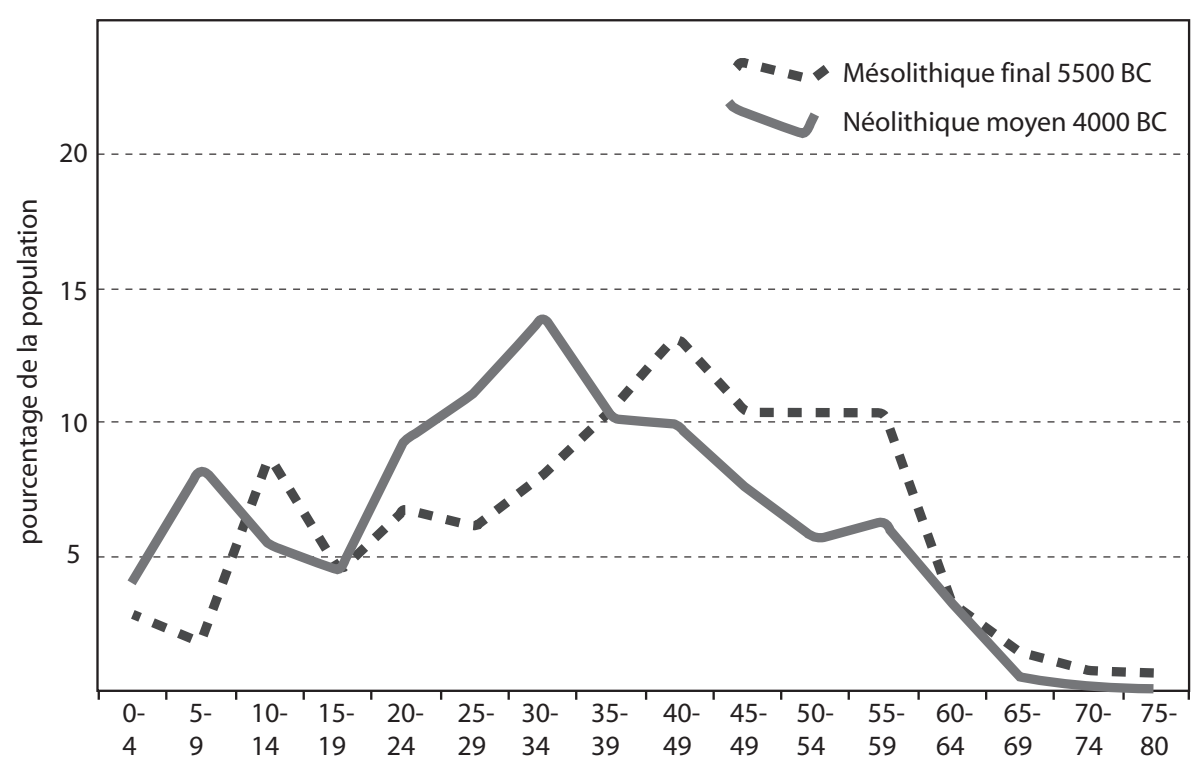

Figure 7 : Estimation de la mortalité au Mésolithique et au Néolithique à partir des nécropoles danoises d'HardinxveldPolderweg et de De Bruin (d'après Smits et Van Der Plicht, 2009).

Figure 7: Estimation of mortality rates during the Mesolithic and Neolithic using data from Danish cemeteries of Hardinxveld-Polderweg and De Bruin (after Smits and Van Der Plicht, 2009). probable de maladies épidémiques aux populations mésolithiques lors de la rencontre des deux cultures a probablement drastiquement réduit cette espérance de vie, si l'on compare à la rencontre historique entre chasseurs-cueilleurs américains et agriculteurs européens du XviII ${ }^{\mathrm{e}}$ au début du $\mathrm{XX}^{\mathrm{e}}$ siècle (Lucas Bridges, 2010 par exemple).

\section{Conclusion}

La difficulté de distinction au Proche-Orient entre les cueilleurs spécialisés du pré-PPNA (ou même du PPNA?) et les premiers agriculteurs est floue. Seule l'absence de céramique dans ces contextes permet de distinguer absolument les derniers mésolithiques des premiers agriculteurs. Mais la présence de céramique en contexte mésolithique en Europe du Nord limite cette possibilité. La distinction entre producteurs et ramasseurs/stockeurs apparait donc davantage comme un trait culturel, voire un interdit (Tchekov, 18907) qui renvoie au concept biblique de "péché originel». La révolution n'est pas néolithique, elle est Holocène et correspond à une adaptation technique de l'homme à un nouveau milieu. Le recours à la culture et à l'élevage n'est qu'une adaptation à une carence en produits par ailleurs naturellement présents dans la nature (céréales, légumineuses, fibres textiles, drogues?). L'adaptation européenne a ainsi lieu globalement dans le "Kurdistan ", région sèche et chaude, et

7. Le Ghiliak considère l'agriculture comme un grand péché : quiconque fouille la terre ou y plante un arbre ne tarde pas à mourir. (L'̂̀le de Sakhaline). n'aurait eu aucun sens autour des grands lacs américains naturellement riches en riz sauvage ou dans l'Europe tempérée croulant sous les glands et les noisettes. L'effet vertueux (ou vicieux?) qui suivit le passage à l'agriculture fut un accroissement démographique très important qui permit en quelques millénaires d'étendre ces techniques de consommation à toute l'Europe. Cependant, avec le passage à la domestication animale, l'homme perd beaucoup : il tombe dans la servitude (Lestel, 2009); il est soumis aux maladies épidémiques qui vont le décimer à plusieurs reprises; il doit produire sa nourriture, ce qui impose un travail pénible. Les peuples de la Bible, lucides, ont résumé cette " déchéance " comme le péché originel, où l'homme est chassé du paradis, mais c'est cette déchéance même qui va lui permettre de croître, de dominer le monde et peut-être à terme de le détruire.

\section{Bibliographie}

Achard-Corompt N., Giros R., Ghesquiere E., Laurelut C., Remy A., Richard I., Riquier V. et SAmson L., à paraître "Premières données sur les implantations mésolithiques fossoyées en Champagne ", in Actes du colloque sur le Mésolithique de Besançon, 2013.

Anderson P. (dir.), I 992 - Préhistoire de l'agriculture. Nouvelles approches expérimentales et ethnographiques, Paris, éditions du CNRS, monographie de CRA n ${ }^{\circ}$ 6, 403 p.

Barbaza M., I999 - Les civilisations postglaciaires, La vie dans la grande forêt tempérée, Paris, La maison des Roches, 128 p. 
Barbier D. et Visset L., 2000 - "La vallée de l'Erve en Mayenne (Massif armoricain, France) a-t-elle joué le rôle de station refuge au cours du dernier épisode glaciaire (Weichsélien) ? ", Life Sciences 323, 2000, p. 469-476.

Billard C., Aubry B., Blancquaert G., Bourhis J.-R., Habasque G., Marinval P., Pinel C. et Ropars A., I995 - « Poses - Le Vivier - Le Clos-Saint-Quentin (Eure) : l'occupation de la plaine inondable au Néolithique et au début de l'âge du bronze ", Revue archéologique de l'Ouest, 1994, nº 11, p. $53-113$.

Bocquet-Appel J.-P., 2009 - « La transition démographique agricole au Néolithique ", in Demoule J.-P. (dir.), La Révolution néolithique dans le monde, Paris, CNRS éditions, 2009, p. 301317.

Bogando A., 2004 - Neolithic Farming in Central Europe. An Archaeobotanical Study of Crop Husbandry Practices, Abingdon, Routledge, 224 p.

Bordes F., I974 - "L'industrie de la grotte ornée de Gouy (SeineMaritime) ", Bulletin de la Société préhistorique française, t. 71, no 4, p. $115-118$.

Cassen S., Boujot C. et Vaquero J., 2000 - Éléments d'architecture, APC Mémoire XIX, 2000, 815 p.

Clutton-Brock J., I 984 - "Neolithic antler picks from Grimes Granves, Norfolk, and Durrington Walls, Wiltshire : a biometrical analysis ", in Excavations at Grimes Graves Norfolk 1972-1976, Fascicule 1, Dorchester, 1984, 55 p.

Colin F.T. et Sturtevant W.C., I 992 - Les Indiens d'Amérique du Nord, Solar, Paris, 256 p.

Crombé P., 20 io - « Early Pottery in Hunter-Gatherer Societies of Western Europe ", in Ceramics before farming, the dispersal of pottery among Prehistoric Eurasian Hunter-Gatherers, Left Coast Press, Walnut Creek, p. 477-498.

Driver H.E. et Massey W.C., I957 - Comparative studies of North American Indians (Transactions 47, Part 2), Philadelphia, American Philosophical Society.

Ghesquiere E. et Marcigny C., 200 I - «Les restes très arasés d'une occupation Néolithique moyen II à Guilberville "La Granchette” (Manche) », Bulletin de la Société préhistorique française, Vol. $98, \mathrm{n}^{\circ}$ 1, p. 77-82.

Ghesquiere E., 20 io - "Une fosse (de stockage?) du Mésolithique récent à Ronai "La Grande Bruyère" (Orne, Basse-Normandie) ", Bulletin de la Société préhistorique française, Vol. 107, nº 3, p. 595-596.

Ghesquiere E. et Aubry B., 2013 - "Mésolithique final et néolithisation en Normandie, carrefour des groupes orientaux et méridionaux", in Transitions, ruptures et continuités en Préhistoire, Jaubert, Fourment et Depaepe (dir.), Actes du XXVII ${ }^{e}$ congrès préhistorique de France, vol. 1 : Evolution des techniques-comportement funéraire-Néolithique ancien, SPF, Paris, 2013, p. 503 à 522.
Gronenborn D., 2010 - «Transregional Culture Contacts and the Neolitization Process in Northern Central Europe ", Ceramics before farming, the dispersal of pottery among Prehistoric Eurasian Hunter-Gatherers, Left Coast Press, Walnut Creek, p. 527-550.

Grootes P.M., Stuiver M., White J.W.C., Johnsen S. et JouZel J., I 993 - Comparison of Oxygen Isotope Records from the GISP2 and GRIP Greenland Ice Cores, Nature 366, p. 552554.

Henon B., Auxiette G. et Ducroce T., 2013 - «Une ou plusieurs fosse(s) du Mésolithique au lieu-dit "Les Etomelles" à Villeneuve-Saint-Germain (Aisne) ", Bulletin de la Société préhistorique française, Tome 110, $\mathrm{n}^{\circ} 4$, p. 751-754.

Jordan P. et Zvelebil M. (ed.), 20 io - « Ex Oriente Lux: The Prehistory of Hunter-Gatherer Ceramic Dispersals ", Ceramics before farming, the dispersal of pottery among Prehistoric Eurasian Hunter-Gatherers, Left Coast Press, Walnut Creek, p. 33-90.

Jordan P. et Zvelebil M. (ed.), 20 Io - Ceramics before farming, the dispersal of pottery among Prehistoric Eurasian HunterGatherers, Left Coast Press, Walnut Creek, 589 p.

Kayser O., Le Goff E. et Roue O., I 990 - «Le site mésolithique de Toul-An-Naouc'h (Plougoulm, Finistère) ", Revue archéologique de l'Ouest, $\mathrm{n}^{\circ} 7$, p. 23-29, 8 fig.

Kilby J.D., 20 i I - « Les caches clovis dans le cadre du Paléoindien ancien en Amérique du Nord ", in Vialou D. (dir.), Peuplements et Préhistoire en Amériques, Paris, CTHS, p. 71-84.

Kroeber Th., I 968 - «Ishi, Testament du dernier indien sauvage d'Amérique du Nord. Terre Humaine ", Plon, [Ishi in two world].

Lestel D., 2009 - « Faire société avec les animaux ", in Demoule J.-P. (dir.), La Révolution néolithique dans le monde, Inrap et Universcience, Paris, 2009, p. 459-472.

Lucas Bridges E., 20 io - Aux confins de la terre, éd. Névicata.

Maigrot Y., 2003 - Étude technologique et fonctionnelle de l'outillage en matières dures animales. La station 4 de Chalain (Néolithique final, Jura, France), Thèse soutenue à l'université de Paris I, mai 2003, 269 p.

MANen C., I 997 - L'axe rhodano-jurassien dans le problème des relations Sud-Nord au Néolithique ancien, BAR S665 (Oxford).

Manen C. et Mazurie de Keroualin K., 2003 - "Les concepts "La Hoguette" et "Limbourg" : un bilan des données ", Cahiers d'archéologie romande 95, ConstellaSion, hommage à Alain Gallay, p. 115-145.

Marchand G., 2009 - Des feux dans la vallée, les habitats $d u$ Mésolithique et du Néolithique récent de l'Essart à Poitiers, Rennes, PUR, 246 p.

Mazurie De Keroualin K., 2003 - Genèse et diffusion de l'agriculture en Europe, Éditions Errance, Paris, 2003, 184 p.

Peteet D., 2000 - « Sensitivity en rapidly of vegetational response to abrupt climate change ", PNAS, 97, 4, p. 1359-1361. 
Philibert S., 2002 - Les derniers "sauvages" - Territoires économiques et systèmes techno-fonctionnels mésolithiques, BAR International Series 1069, 193 p.

Rostain S., 20 I I - "Le peuplement précolombien de l'Amazonie ", in Vialou D. (dir.), Peuplements et préhistoire en Amériques, Paris, CTHS, p. 331-343.

Rozoy J.-G., I 999 - Le mode de vie au Mésolithique. L'Europe des derniers chasseurs, Epipaléolithique et Mésolithique, Actes du $5^{\mathrm{e}}$ Colloque International UISPP, Commission XII, Grenoble, 18-23 septembre 1995, Paris, ed. du CTHS, p. 39-51.

Smits E. et Van Der Plicht J., 2009 - Mesolithic anc Neolithic human remains in the Netherlands: physical anthropological and stable isotope investigations, Journal of Archaeology in the low countries, 1-1 (May 2009).

Stuiver M., Pieter M. et Grootes P.M., 2000 -The GISP2 B18O climate record of the past 16,500 years and the role of the sun, ocean, and volcanos, Quaternary Research, 44, p. 341 à 354 .

Tснекоv A., I 890 - Lîle de Sakhaline, [réed. 2001], Paris, Gallimard, coll « Folio Classique».
TESTART A., I982 - Les chasseurs-cueilleurs ou l'origine des inégalités, Paris, Société d'Ethnographie, 1982, 255 p.

Testart A., 2000 - «Que peut dire aujourd'hui l'anthropologie sociale des chasseurs-cueilleurs d'hier? ", in Les derniers chasseurs-cueilleurs d'Europe occidentale (13000-5500 av. J.-C.), Actes du Colloque international de Besançon (Doubs, France), 23-25 octobre 1998, Presses universitaires franc-comtoises, Besançon 2000, p. 343-350.

Vanmontfort B., Kooijmans L. L., Amkreutz L. et Verhart L., 20 Io - Pots, Farmers and Foragers, Archaeological Studies Leiden University 20, Amsterdam, 2010, 213 p.

Verjux C., 2000 - «Les fosses mésolithiques d'Auneau (Eureet-Loir - France) ", in Méso '97, Actes de la Table-ronde "Epipaléolithique et Mésolithique ", Lausanne, 21-23 novembre 1997 (P. Crotti éd.), Cahiers d'Archéologie romande, $\mathrm{n}^{\circ} 81$, p. $129-138$.

Verjux C., 2004 - «Creuser pour quoi faire ? Les structures en creux au Mésolithique ", in Approches fonctionnelles en Préhistoire (Bodu et Constantin [dir.]), XXV ${ }^{\mathrm{e}}$ Congrès préhistorique de France, Nanterre 24-26 novembre 2000, 2004, p. 239-248.

Zusammenfassung: Einige Überlegungen zu den Lebens- und Umweltverbältnissen im Meso- und Neolithikum - Ab etwa 15000 BP beobachtet man eine allgemeine globale Erwärmung und in insbesondere in Frankreich eine gravierende Veränderung der Klimaverhältnisse. Diese Veränderungen sind spektakulär: Im Nordteil Frankreichs, der bisher von einer steppenartigen, offenen Vegetation eingenommen wurde, kommt es zur Wiederbewaldung. Die spätglaziale Fauna wird durch Hirsche, Rehe und Wildschweine abgelöst. Der Mensch, der zu spätglazialen Zeiten die Gebiete nur vereinzelt aufgesucht hatte, siedelt sich nun dauerhaft in den verschiedenen Landschaftstypen an. Bis vor kurzer Zeit nahm man an, dass die mesolithische Bevölkerung sehr verstreut lebte und sich vorwiegend von der Jagd ernährte, so wie die letzten noch heute existierenden Jäger-Sammler-Gesellschaften in der Welt, die in wüstenartigen Lebensräumen existieren (Pygmäen, Luits, Buschmänner...). Es erscheint nun dringend geboten, diese überkommene Vorstellung des "guten Wilden" zu beenden und durch diejenige gut strukturierter Bevölkerungsgruppen zu ersetzen. Diese neuen Überlegungen beinhalten auch eine weitgehend diversifizierte Ernährung und eine Sesshaftigkeit der Bevölkerung, möglicherweise begleitet von mehr oder weniger gewaltsamen Konflikten benachbarter Bevölkerungsgruppen.

Resumen: Elementos de reflexión sobre las economías mesoliticas y neoliticas - Desde -15.000 BP, se observa en el mundo en general y en Francia en particular, un cambio climático. Las mudanzas son espectaculares: la mitad del norte de Francia, ocupada en este momento por la estepa, fue reconquistada por la vegetación arbórea. La fauna característica del frío se sustituye por los ciervos, corzos y jabalies. El hombre que, durante el periodo glacial sólo ocasionalmente ocupaba el territorio, se traslada de forma permanente en todos los hábitats. Hasta hace poco se pensaba que las poblaciones del Mesolitico eran muy dispersadas, que vivian principalmente de la caza, como lo practican aun las últimas civilizaciones de cazadores-recolectores en el mundo que operan en ambientes desérticos (Pigmeos, Inuit, hombres del Bush...). Ya es hora de poner fin a esta imagen pesimista del buen salvaje y proponer una visión de sociedades organizadas. Los nuevos elementos de reflexión sugieren una dieta ampliamente diversificada y una población sedentaria, probablemente acompañado de un estado de violencia entre los grupos vecinos más o menos importante.

Schlüsselwörter: Mesolithikum, Klima, Holozän, Eicheln, Haselnüsse, Getreide, Domestikation, Territorium, Konservierung, Wirtschaft, Lebenserwartung.

Palabras clave: Mesolitico, clima, Holoceno, bellotas, frutos secos, cereales, domesticación, territorio, conservación, economía, esperanza de vida. 